\title{
Musical B-boying: \\ A Wearable Musical Instrument by Dancing
}

\author{
Minoru Fujimoto ${ }^{1}$, Naotaka Fujita ${ }^{1}$, Yoshinari Takegawa ${ }^{2}$, \\ Tsutomu Terada ${ }^{1}$, and Masahiko Tsukamoto ${ }^{1}$ \\ ${ }^{1}$ Graduate School of Engineering, Kobe University \\ ${ }^{2}$ Organization of Advanced Science and Technology, Kobe University \\ \{minoru,nfujita\}@stu.kobe-u.ac.jp, \{take,tsutomu\}@eedept.kobe-u.ac.jp, \\ tuka@kobe-u.ac.jp
}

\begin{abstract}
Advances in computer technologies have enabled new means of musical expression with motion enabled musical instruments, which have attracted a great deal of attention. We created a new performance system that enables a dancer to make music while b-boying, which is a dance style. We implemented sensor-equipped shoes and a system that recognizes motions and that controls music. We used an implemented prototype on stage, and we found that dancers were able to control the music flexibly while b-boying. Our system will bring about a new style of musical performance for both musicians and dancers.
\end{abstract}

\section{Introduction}

Advances in computer technologies have enabled new means of musical expression with motion enabled musical instruments, which have attracted a great deal of attention 1 2, 3] 4]. These instruments enable users to generate and control the sound by physical motion, and they have led to the development of a new style of entertainment that has the characteristics of integration between physical performance and musical performance. Although a lot of research and art performances have such characteristics, they does not fulfill the requirement that players want to keep the procedure of whole performance under their control. We created a new dance performance system that enables dancers to generate music while dancing. We implemented sensor-equipped shoes and a music control system with these shoes. Our system not only generates sounds when people dance, but it also controls the performance scenario by changing the set of sounds generated by motion commands. Using our system, dancers can flexibly control sound generation while dancing, thereby enabling a new style of musical performance for both musicians and dancers.

\section{B-BOYING}

B-boying is commonly known as "breakdancing", which is a type of street dance. It consists of four elements: entry, footwork, power moves, and freeze. Entry refers to upright dancing. Footwork is a dance performed on the floor. A power 
move is the most impressive performance because it includes acrobatic moves. A freeze is a stylish pose in reaction to certain beats and is also used to end a performance.

Combining these techniques allows for an infinite number of expressions. Moreover, the dancer adds soul to his/her routine by using various dancing motions including delicate hand-movements and dynamic movements using the whole body.

In addition to the dancing techniques, B-boying has two types of performance styles: showcases and dance battles. In showcase style, people dance to the music. However, in a dance battle, they face each other and compete in turns with improvised performances. Each player has to change his/her expression dynamically in response to the music and the movement of opponents.

\section{System Design}

As stated previously, b-boying has an infinite range of expression. Additionally, if the dancers were able to generate/control sound with their dancing, a new style of performance would emerge. An integration of dance and sound creation would allow dancers to control everything on stage, and the quality of their performance would improve. Moreover, dancers in dance battles would need dancing and musical skills, raising the interest in dance battles. Therefore, we propose a new style of performance where dancers control not only their motions but also sound generation. In addition to this, if the system has a function to change the allocation between motions and sounds by the dancer's actions, they can fully control the performance on their own.

\subsection{Requirements}

In usual dancing performances, we dance to the music passively. Dancers or choreographers statically compose the choreography considering the flow and tempo of the music. The configuration of dancing is static, and changing the configuration in response to problems and atmosphere is difficult.

Our system enables dancers to control their performance dynamically by changing the sound generated while dancing. Our pilot study enabled us to clarify these system requirements:

No restriction. The equipment must be small and light enough so as not to restrict movement to enable the quality of dance to remain high. In particular, the devices must be wireless.

Motion flexibility. To play/control music with movements, the system should allow users to configure the relationship between a dance step and a phrase flexibly. Moreover, various types of motions should be accepted by the system.

Personalization. There are various styles of dancing, and motions vary among individuals. Therefore, the motion detection mechanism should be personalized for each user. 

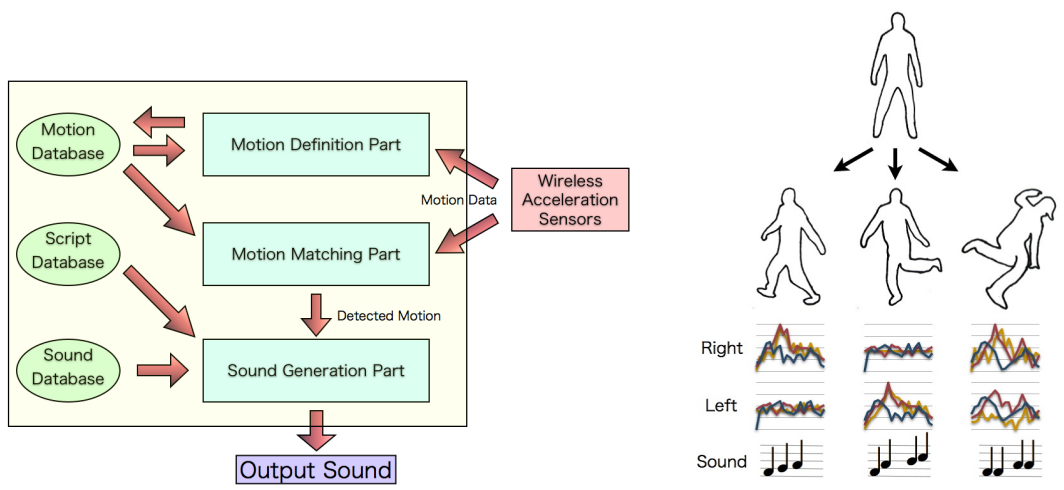

Fig. 1. System structure

Fig. 2. Example of motion detection

Configuration flexibility. A dance showcase is a kind of story. Therefore, the system should support a change in setting. The system should enable dancers to change a set of motions and allocated sounds according to the change in the setting.

\subsection{System Structure}

Figure 1 shows the system structure of our system. It consists of 3-axis wireless acceleration sensors equipped in both shoes and a PC application for processing acquired sensor data and for generating sounds. Because dancers only have to wear small sensors on their shoes, the system does not interfere with their dancing (No restriction). Our system uses feet motions to recognize dancers' actions. This is because b-boying has many different characteristics in the way dancers move their feet.

The flow of our system is as follows:

Step0. Before using the system on stage, the user registers motions and scripts.

Step1. While a performer dances, the motion of his/her feet is continuously transmitted to the system on a PC.

Step2. The system recognizes the motion by comparing the acquired motion and stored motion data from a motion database.

Step3. By retrieving actions allocated to the recognized motion using a script database, the system decides the action to take such as generate sounds or change the set of commands/sounds.

When dancers first use our system, they register their motions in it. To register a motion, a dancer performs the actual motion while wearing the acceleration sensors on his/her shoes. This creates gmotion flexibilityh and gpersonalizationh because the user can register any motion freely. Then, the dancer describes the scripts. A script includes various definitions of mappings between motions 
Table 1. Commands used in script

\begin{tabular}{c|l}
\hline \hline Name & Function \\
\hline right $a c t$ & recognition of act on right foot \\
left act & recognition of act on left foot \\
both $a c t$ & recognition of act on both feet \\
set $g r p$ & creation of a command group grp \\
play snd & specification of the sound snd to play \\
change to $g r p$ & jump to a command group grp \\
for $n$ times & $n$ times recognition is required \\
after act & sequential recognition after act \\
\hline
\end{tabular}

set block1 ...(1)

right Sample1 play Music1 ...(2)

left Sample1 change to block2 ...(3)

set block2

both Sample1 for 3 times play Music2...(4)

left Sample1 after left Sample2 play Music3...(5)

right Sample1 change to block1

Fig. 3. Example of script description

and commands, such as generating sounds, changes in music, and changes in a mapping set (the detailed functions in a script are described in the next section). It contributes to the Configuration flexibility of the system.

When used on stage, the wearing sensors continuously transmit the motion data to the PC application via wireless communication. The system recognizes the motion by comparing the acquired motion and stored motion data from the motion database. Our system uses a dynamic programming (DP) matching algorithm to analyze the acceleration-sensor data. DP matching is a well-known method for retrieving similarities in time series data such as speech or motions. Because each sensor outputs 3-dimensional acceleration data, the system acquires 6-dimensional data for comparison.

The system retrieves the actions allocated to the detected motion using the script database. For example, Figure 2 shows the generation of three types of sounds according to three different motions.

\subsection{Script for Dancing}

To achieve flexibility, we use a scripting mechanism for enabling dancers to change the system settings as they like. Our script consists of a set of commands, which are shown in Table 1.

Figure 3 shows an example of a script description. Each part of the figure means the following functions:

1. Definition of a command group block1: this group continues until another group definition. 


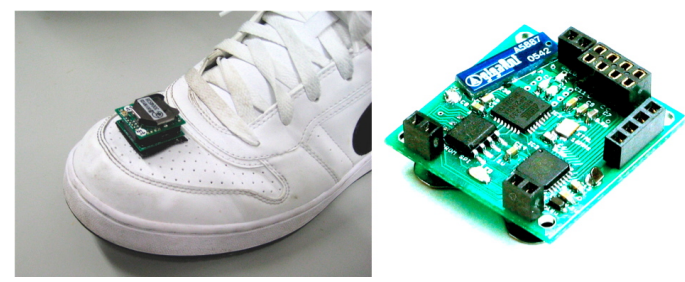

Fig. 4. Acceleration sensor with shoes

2. The system outputs Music1 when Sample1 motion is recognized on the right foot.

3. The system changes the group to block2 when Sample1 motion is recognized on the left foot.

4. The system outputs Music2 when Sample1 motion is recognized on both feet three times in succession.

5. The system outputs Music3 when Sample1 motion is recognized on the left foot sequentially after the recognition of Sample2 on the left foot.

These functions enable a dancer to utilize the system flexibly. In particular, the group definition function contributes to the flexibility and accuracy of recognition. This function enables the system to allocate different sounds to one motion, and because unnecessary motions are excluded for recognition, the recognition accuracy increases compared with a case where all motions are recognized.

An example of using the grouping function on an actual stage is a dancer playing drum-music in the first half of the show, then playing bass-music in the second half.

\section{Implementation}

\subsection{Hardware Implementation}

As described in Section 3.1, we needed small wireless 3-dimensional acceleration sensors on dancing shoes. No suitable sensor was available in commercial products, so our research group developed a new wireless sensor module named the "Nao-RF Chip". This module has a 70Hz 3-dimensional acceleration sensor, and the size and weight allow it to be attached to shoes.

Figure 4 shows the sensor module and a shoe with the module.

\subsection{Performance}

We used our system in several actual dance performances. One example of such performances was a showcase at the Kobe Luminarie Live Stage on December 8th and 9th, 2007. Kobe Luminarie is a light festival held in Kobe, Japan every December. It began in 1995 and commemorates the Great Hanshin earthquake of that year. 

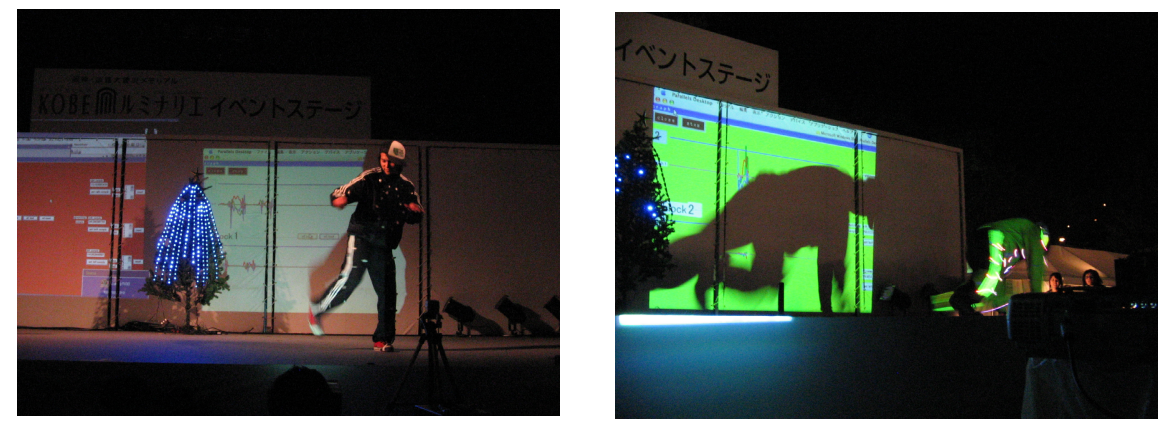

Fig. 5. Snapshot in Kobe Luminarie (1) Fig. 6. Snapshot in Kobe Luminarie (2)

Our show consisted of various types of dancing, as shown in Figure 5, 6, In the show, two dancers improvised performances in turn. Using our system, they changed the music pattern while dancing. Moreover, because we added a function to change the projected background color in response to motion, the audience became more interested in the performance.

\section{Conclusions}

We described a new style of system that enables dancers to make music while they dance. Our system uses a scripting mechanism to control performances flexibly. We focused on a person-centered design where users can customize everything, and this characteristic contributes to enabling their emotions to be expressed intuitively and to creating a new type of entertainment. Actual use of the system on stage revealed that dancers could effectively make music.

In the future, we will create an algorithm with high recognition accuracy. In addition, we will apply our system to various types of dancing styles other than b-boying.

\section{References}

1. Aylward, R., Paradiso, J.: Sensemble: A Wireless, Compact, Multi-User Sensor System for Interactive Dance. In: Proc. of the International Conference on New Interface for Musical Expression, pp. 134-139 (2006)

2. Bromwich, M., Wilson, J.: 'BODYCODER' A sensor suit and vocal performance mechanism for real-time performance. In: Proc. of the International Computer Music Conference, pp. 292-295 (1998)

3. Tanaka, A.: Musical Technical Issues in Using Interactive Instrument Technology with Application to the BioMuse. In: Proc. of the International Computer Music Conference, pp. 124-126 (1993)

4. YAMAHA MIBURI (1990), http://www.yamaha.co.jp/design/products/1990/miburi/ 